\title{
TRANSPORT PROPERTIES OF SILICON $\delta$-DOPED GaAs IN HIGH ELECTRON DENSITY REGIME
}

\author{
A.V. Buyanov, P.O. Holtz, G. Pozina, B. Monemar \\ Department of Physics and Measurements Technology, Linköping University \\ 581 83, Linköping, Sweden
}

\section{J. Thordson and T.G. Andersson}

Department of Physics, Chalmers University of Technology and Göteborg University 412 96, Göteborg, Sweden

\begin{abstract}
We report results for $\mathrm{Si}$ layers embedded in $\mathrm{GaAs}$, extending from the delta-doped ( $\delta$-doped) range up to 6 monolayers derived by means of variable temperature resistivity and Hall effect measurements, secondary ion mass spectrometry and high resolution X-ray diffractometry techniques. The conductivity transition from free carrier transport in ordered $\delta$-layers $(<1 \mathrm{ML})$ to strongly-localized two-dimensional variable range hopping transport under potential fluctuation disordered conditions ( $>4 \mathrm{ML}$ ) is clearly observed. This observation is in good agreement with the secondary ion mass spectrometry and high resolution X-ray diffractometry data. Results from the intermediate case with 2-3 MLs are also discussed.
\end{abstract}

PACS numbers: $61.72 . \mathrm{Vv}, 68.55 . \mathrm{Ln}, 72.60 . \mathrm{gg}$

A thin layer of $\mathrm{Si}$, say one or a few atomic layers, introduced into the GaAs lattice will exhibit properties, which should be scaled down to the ultimate spatial limit in the growth direction. The thickness of such an Si layer is less than other relevant length scales, e.g. the de Broglie wavelength, and is sufficiently narrow to mathematically be described by Dirac's delta function in the ideal case. Such an Si layer can accordingly be ascribed as a $\delta$-layer. With the described $\mathrm{GaAs} / \mathrm{Si} / \mathrm{GaAs}$ system, we consequently encounter an interesting system for investigations of low-dimensional properties. The system, involving a dissimilar III-V/IV heterostructure constitutes, however, a growth challenge. There are many limitations for the practical use of $\delta$-layers in device structures, such as saturation in the free carrier density as well as migration of Si-atoms away the ideal $\delta$-plane. In order to reduce the effect of these limiting factors extensive investigations are required. The realization of the $\delta$-structure also has potential applications, such as photonic devices and as a source to achieve high electronic sheet densities in various electronic devices [1]. 
Previous high energy electron diffraction (RHEED) investigations of thin $\mathrm{Si}$ layers in GaAs have shown that 3D islands are formed for Si layers thicker than $3 \mathrm{MLs}$ [2]. With cross-section transmission electron microscopy (XTEM), defects such as twins [3], stacking faults [2], and misfit dislocations [3] due to relaxation occurring already at $2 \mathrm{MLs}$ have been observed. Still the Si layers are well accommodated, giving only a modest degradation of the cap-layer crystallinity $[2,4]$. The critical layer thickness for pseudomorphic growth have been calculated with results in a wide range, 19-69 MLs [5-8]. All these values are clearly higher than the experimental results, indicating that the reliability of the models used at this large lattice mismatch (4.1\%) is limited, since these models are not intended for atomic scale layer thicknesses but derived from bulk elasticity theory.

In this letter we report on variable temperature resistivity and Hall effect measurements in GaAs structures having up to $6 \mathrm{Si} \mathrm{MLs,} \mathrm{aiming} \mathrm{at} \mathrm{a} \mathrm{better} \mathrm{under-}$ standing of carrier transport properties. The transition from low-field free carrier transport in the ordered Si $\delta$-doped layers embedded in GaAs (up to $1 \mathrm{ML}$ ) to high temperature strongly localized two-dimensional variable range hopping (2D-VRH) transport in disordered layers (more than $4 \mathrm{MLs}$ ) is revealed. The transport measurements are found to be in good quantitative agreement with the high resolution X-ray diffractometry (HRXRD) and secondary ion mass spectrometry (SIMS) data.

The structures were grown with a solid arsenic source in a Varian GEN II modular system on In-mounted semi-insulating GaAs (100) substrates, and with $\mathrm{Si}$ deposited from a dopant source. The exposure time of $\mathrm{Si}$, with the cell at $1300^{\circ} \mathrm{C}$ (thermocouple temperature), was several hours $(\approx 2 \mathrm{~h} / \mathrm{ML})$. At the end of the $0.5 \mu \mathrm{m}$ thick GaAs buffer layer growth, the substrate temperature was reduced from $580^{\circ} \mathrm{C}$ to $500^{\circ} \mathrm{C}$. Both the Si layer and the $100 \mathrm{~nm} \mathrm{GaAs}$ cap-layer were grown at $500^{\circ} \mathrm{C}$.

The transport studies were performed within the temperature range $1.5-300 \mathrm{~K}$ in a superconductor $16 \mathrm{~T}$ solenoid magnet. Hall measurements were done in an applied magnetic field up to $0.3 \mathrm{~T}$. All transport data presented in this paper were taken by a low-amplitude dc-current (1-7 $\mu \mathrm{A})$ technique. The resistivity measurements were performed in the dark on lithographically defined Hall bars with $\mathrm{Au} / \mathrm{Ge} / \mathrm{Au}$ ohmic-contacts. The Van-der-Pauw geometry was used for Hall effect measurements. HRXRD measurements were performed on the samples in order to provide a qualitative judgment about the layer crystallinity. SIMS studies were carried out in order to obtain information about the Si diffusion from the doping plane.

The variable temperature resistivity data obtained for the $\delta$-doped structures are presented in Fig. 1. The results are quoted in terms of the sheet resistivity for the Si-plane in GaAs. It is clear from Fig. 1 that the resistivity, $\rho$, for the $1 \mathrm{ML}$ sample is practically temperature independent within the entire temperature range, indicating "degenerate conductivity" for a free electron gas. The 4 and 6 MLs samples, on the other hand, demonstrate the temperature dependence, $\rho \sim \exp \left(T_{0} / T\right)^{1 / 3}$, known as Mott's law for a noninteracting 2D carrier gas in the VRH-regime [9]. Taking into account that the 4 and 6 MLs samples are degenerate $[10,11]$, the observation of such a temperature dependence of the conductivity in 


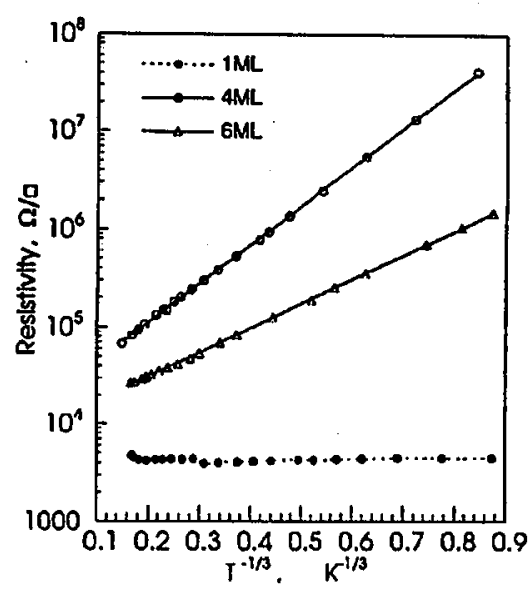

Fig. 1. The sheet resistivity vs. $T^{-1 / 3}$ (Mott's law for 2D-VRH transport) for strongly-localized transport (4 MLs and $6 \mathrm{MLs}$ samples). The $1 \mathrm{ML}$ sample demonstrates "degenerate conductivity" for a free electron gas.

the degenerate system is quite remarkable. First of all, the 2D-hopping conduction is usually observed below the metal-insulator transition after the formation of the impurity band. At low temperatures, only the presence of residual acceptors (for $n$-type material) can create vacant positions in the impurity band, because some small compensation has already taken place and gives rise to 2D-hopping conductivity in the $\delta$-plane. Above the degenerate limit, the electron subbands are partly filled and the low-temperature conductivity is usually metal-like [12]. The experimental observation of hopping transport provides evidence for strong carrier localization. To estimate the localization length $\xi$ of the electron wave function, we have used the known Mott equation, $k T_{0}=3.5 /\left[g\left(E_{\mathrm{F}}\right) \xi^{2}\right]$, where $g\left(E_{\mathrm{F}}\right)$ is the density of states at the Fermi level. The estimated values are as follows: $T_{0}=780 \mathrm{~K}, \xi=150 \AA$ for the $4 \mathrm{MLs}$ sample and $T_{0}=190 \mathrm{~K}, \xi=151 \AA$ for the $6 \mathrm{MLs}$ sample. The density of states $g\left(E_{\mathrm{F}}\right)$ was assumed to be energy independent [13].

In Fig. 2a the data from the SIMS analysis are shown. For samples with 3 MLs or less, the peak width is constant and is limited by the SIMS resolution 40-50 $\AA$. The integrated peak area scales the amount of $\mathrm{Si}$ in the layer. For the 4 and 6 MLs samples, there is a substantial broadening of the peaks. The peak width increases to a value of the order 160-250 $\AA$ (a factor of 4-5 above the SIMS depth resolution). This value is comparable with the localization length $\xi \approx 150 \AA$ for both 4 and 6 MLs structures. These results imply that the observed strong localization of carriers are induced by the degradation of the Si-doped layer, to highly disordered layer. Further evidence for the disorder at a width of 4 MLs is provided by the HIRXRD measurements shown in Fig. $2 \mathrm{~b}$. The satellite fringes can be seen in the 1 and 3 MLs samples, but have almost disappeared in the 4 MLs sample, again demonstrating the degradation of the crystallinity. 

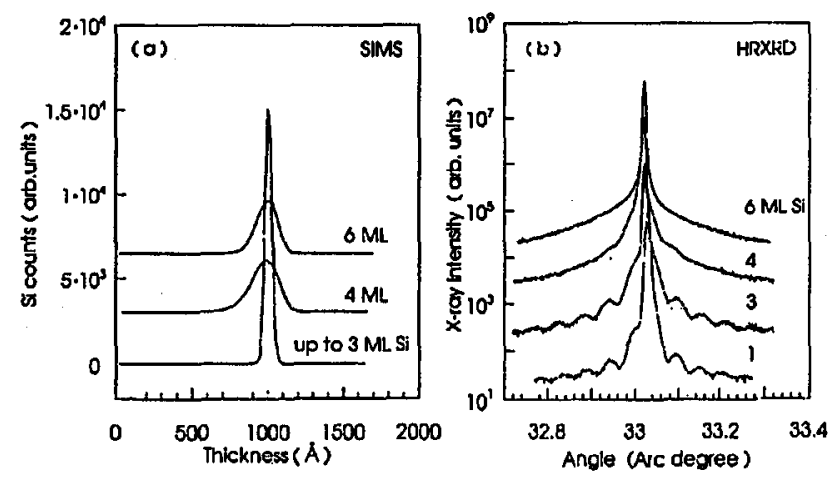

Fig. 2. (a) Secondary ion mass spectra from 3,4 and 6 ML samples showing that the $\mathrm{Si}$ diffusion is limited (below the SIMS resolution) up to $3 \mathrm{MLs}$, while significant $\mathrm{Si}$ diffusion is seen in the 4 and 6 MLs samples. (b) High resolution diffraction spectra. The satellite fringes are almost absent for Si layers thicker than $4 \mathrm{MLs}$, but are clearly seen in sample with less than 3 MLs.

It should be noted that the observed temperature range for the VRII-conductivity is quite unusual. High temperature hopping has so far only been reported for low-doped $p$-type bulk GaAs grown at low temperature [14, 15], and has been attributed to strong fluctuations of the potential. Considering that the localization of the wave function of electrons coincides with the geometrical localization of the dopant spike, a strong influence of the potential fluctuations due to the disorder on the transport properties of the 2D electron gas (2DEG) is expected. Thus we propose that the main mechanism of the observed high-temperature 2D-VRH transport is the strong localization of the 2DEG due to the disorder related potential fluctuations.

It should be pointed out that the low-temperature 2D-VRH transport in dilute Si $\delta$-doping layers in GaAs is well known [12]. This strongly localized transport has been experimentally and theoretically investigated in structures with the Si-doping level below the degenerate limit and has been described according to the impurity band principles $[9,12]$. In the present letter we propose 2D-VRH transport in $\mathrm{Si} \delta$-doping layers in $\mathrm{GaAs}$, for $\mathrm{Si}$ doping levels, which are several orders of magnitude higher than the degenerate limit $\left(\approx 10^{11} \mathrm{~cm}^{-2}\right.$ for this system [12]). The 2D-VRH transport observed in our study occurs at higher temperatures and is caused by strong potential fluctuations due to the disorder.

We will finally point out that the variable temperature Hall effect data obtained for GaAs with 2-3 MLs Si i.e. an intermediate case is more complicated. As can be seen in Fig. 3, the measured sheet concentration exhibits a striking temperature dependence. The sheet concentration is drastically reduced when the temperature increases from $\approx 50 \mathrm{~K}$ up to room temperature. This clearly deviates from what is observed for the $1 \mathrm{ML}$ sample, also shown in Fig. 3 for comparison, where the sheet concentration is temperature independent, as characteristic of a low-field free-electron transport. This is despite the fact that the SIMS and 


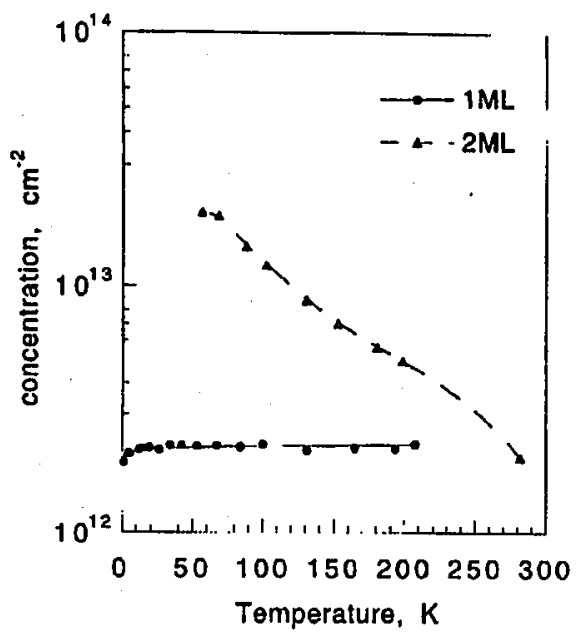

Fig. 3. The variable temperature Hall data for an intermediate case with 2 MLs. The corresponding Hall data for the $1 \mathrm{ML}$ structure is shown, exhibiting an almost temperature independent sheet concentration.

HRXRD data show no marked difference between these two samples in the Si profile and crystallinity. The mechanism responsible for the behaviour deduced from the Hall data for the 2 MLs sample is still unknown. A similar temperature dependence of the carrier concentration has previously been reported by Look et al. [14] and was explained as a result of the simultaneous contribution of hopping and free carrier conduction. The 3 MLs structure exhibits a very high resistivity, different from the other samples. The reason for that is still unclear and requires further investigation.

In conclusion, we have studied $\mathrm{Si}$ layers, embedded in GaAs, extending from the $\delta$-doping range up to $6 \mathrm{MLs}$ by means of variable temperature resistivity and Hall effect measurements, SIMS and HRXRD techniques. The free carrier delocalized transport prevails in the ordered $\delta$-layers $(<1 \mathrm{ML})$, while strongly-localized 2D-VRH transport under potential fluctuation disorder conditions clearly observed for wider Si-layers (> $4 \mathrm{ML}$ ). This observation is in good agreement with the SIMS and HRXRD data. The properties of Si dopant layers in the intermediate range (2-3 MLs) deviate, however, and further studies are needed to understand their properties in more detail. We want to thank Dr. Ulf Södervall for the performing of the SIMS measurements.

\section{References}

[1] S.E. Ralph, F. Capasso, R.J. Malik, Appl. Phys. Lett. 57, 626 (1990).

[2] K. Adomi, S. Strite, H. Morkoç, Y. Nakamura, N. Otsuka, J. Appl. Phys. 69, 220 (1991).

[3] T. Sudersena Rao, Y. Horikoshi, C. Jagadesh, R.G. Elliman, J.S. Williams, Jap. J. Appl. Phys. 31, 3282 (1992).

[4] D.J. Liu, J.C. Fan, C.P. Lee, K.H. Chang, D.C. Lion, J. Appl. Phys. 73, 608 (1993). 
[5] H. Tanino, S. Amano, H. Kawanami, H. Matsuhata, J. Appl. Phys. 70, 1978 (1992).

[6] J.H. van der Merwe, J. Appl. Phys. 34, 117 (1963).

[7] J.W. Mattews, A.E. Blakeslee, J. Cryst. Growth 27, 118 (1974).

[8] R. People, J.C. Bean, Appl. Phys. Lett. 47, 322 (1985).

[9] N.F. Mott, G.A. Davis, Electronic Processes in Non-Crystalline Materials, Clarendon, Oxford 1979.

[10] J.V. Thordson, P. Songpongs, G. Swenson, T.G. Andersson, J. Cryst. Growth 150, 696 (1995).

[11] J.V. Thordson, Lic. Thesis, Chalmers University of Technology, Göteborg 1995.

[12] Q. Ye, B.I. Shklovskii, A. Zrenner, F. Koch, K. Ploog, Phys. Rev. B 41, 8477 (1990); E.I. Levin, M.E. Raikh, B.I. Shklovskii, Phys. Rev. B 44, 11281 (1991).

[13] C. Johnson, H. Jiang, Phys. Rev. B 48, 2823 (1993).

[14] D.C. Look, D.C. Walters, M.O. Manasreh, J. Sizelove, C. Stutz, K. Evans, Phys. Rev. B 42, 3578 (1990).

[15] Kai Zhang, D. Miller, J. Electron. Mater. 22, 1433 (1993). 